\title{
EDUCACIÓN CÍVICA Y LA CONSTRUCCIÓN DE LA CIUDADANÍA
}

\section{CIVIC EDUCATION AND THE MAKING OF CITIZENSHIP *}

\author{
Erika Tapia Nava ${ }^{1}$
}

México

Palabras Clave: Ciudadanía, Educación cívica, democracia, Cultura política.

Keywords: citizenship, civic education, democracy, political culture.

\section{RESUMEN}

En este articulo se brindan los antecedentes filosóficos de la educación cívica y se abordan las dos corrientes teóricas que predominan en la educación cívica: el liberalismo y el comunitarismo cívico. A partir de ello, se desarrolla una reflexión sobre lo que puede ser la educación cívica y su relación con la noción de ciudadanía. La propuesta consiste en que la ciudadanía no debe ser vista únicamente como una condición jurídica, sino que debe apoyarse en una serie de valores que le den sustento para que la democracia se desarrolle no sólo como una forma de gobierno sino también como una forma de vida, la cual promueva la cultura democrática.

\section{ABSTRACT}

This paper provides philosophical background of civic education and addresses the main two theoretical approaches in civic education: liberalism and civic

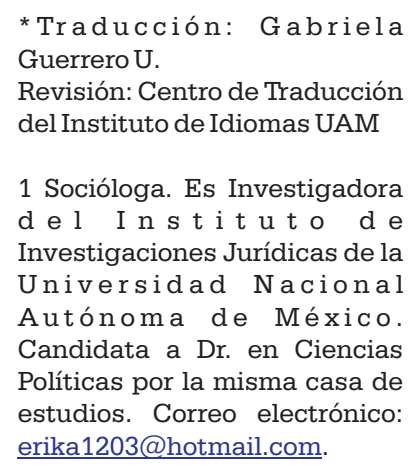

1 Socióloga. Es Investigadora d e l I n s t i t u t o d e Investigaciones Jurídicas de la Universidad Nacional Autónoma de México. Candidata a Dr. en Ciencias Políticas por la misma casa de estudios. Correo electrónico: erika1203@hotmail.com. 
communitarism. Based on them, a reflection on what civic education can be and its relation with the concept of citizenship is developed. The proposal is to conceive citizenship not only as a merely judicial condition, but also be supported by values supporting the democracy to be developed as a form of government or as a lifestyle promoting democratic culture.

\section{INTRODUCCION}

Los antecedentes filosóficos de la educación cívica provienen de las ideas de los griegos y los romanos, quienes desarrollaron las primeras experiencias de ciudadanía. Pero su antecedente más inmediato fue la discusión que se desarrolló durante la Ilustración. En el siglo XVIII, el "siglo de las luces", se constituyó la ciudadanía moderna que incorporó principios universalistas expresados en los derechos individuales, la participación política, el sufragio universal y la educación para los ciudadanos. Sin embargo, la noción de ciudadanía se ha transformado con los procesos históricos, por lo que podríamos decir que la ciudadanía es un proceso dinámico mutable e inacabado.

Desde una perspectiva genérica amplia, la ciudadanía es un estado civil que supone elementos jurídicos, políticos y morales. Identifica a aquellos miembros de una comunidad política o Estado que han de estar protegidos por las instituciones y al mismo tiempo, están dispuestos a contribuir en ellas. En el ámbito jurídico, la ciudadanía es un título que sirve para reconocer la pertenencia de una persona a un Estado y su capacidad individual como miembro activo de éste. Es decir, la ciudadanía equivale al reconocimiento de una serie de derechos y deberes, relacionados con la participación en la esfera pública. La ciudadanía lejos de ser mera adquisición de derechos y obligaciones, constituye una cualidad moral: se trata de una cualidad distintiva; el hecho de pertenecer a una comunidad política. ${ }^{2}$

De acuerdo con la tradición republicana, el ejercicio de la ciudadanía requiere educación. Es decir, ser ciudadano exige, fundamentalmente, una actuación o práctica, y no simplemente el reconocimiento de determinados derechos. Esta práctica debe ser cultivada a través de una educación basada en el ejercicio de las virtudes cívicas ${ }^{3}$ Por lo tanto, la educación cívica es la acción y efecto de desarrollar las facultades morales e intelectuales pertenecientes o relativas al
2 Fernando Bárcena, El oficio de la ciudadanía, Paídos, Buenos Aires, 1997, p. 152.

3 bid., p. 149.

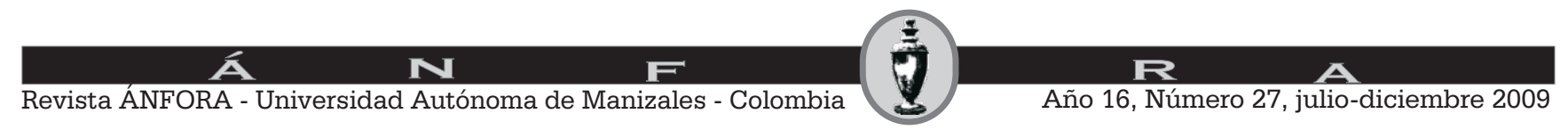


ciudadano. ${ }^{4}$ Ésta se constituye como una condición facilitadora de una democracia fuerte.

\section{El liberalismo y comunitarismo en la educación cívica}

La concepción de ciudadanía durante muchos años tuvo poca atención y como noción del discurso político fue notablemente escasa. Sin embargo, en el transcurso de los últimos treinta años, las discusiones de filosofía política se han articulado en torno a tres nociones: justicia, comunidad y ciudadanía, mismas que constituyen tres aspectos fundamentales de la educación cívica. Ello ha provocado la aparición y desarrollo de una serie de debates, sobre todo a partir de los años ochenta ${ }^{5}$

Estos debates han sido protagonizados por los liberales y comunitaristas sobre todo de Norteamérica. Los debates incluyen dos clases de referencias teóricas fundamentales: 1) aquellas relacionadas con la disputa moral en torno a la ética del deber y la ética de la virtud y 2) aquellas relativas a la naturaleza de la democracia y de la ciudadanía. ${ }^{6}$

En los Estados Unidos el término liberal alude principalmente a una posición aunque un poco indefinida de izquierda o progresista. En el ámbito académico posee una connotación plural, llena de matices, hasta convertirse en una línea de pensamiento en la que se sitúan muchas posiciones teóricas ${ }^{7} \mathrm{El}$ supuesto prestigio del liberalismo, está avalado por distintas razones, por un lado, se debe a los éxitos prácticos del ofrecimiento de bases normativas y organizativas relativamente coherentes entre sí, las cuales se han mostrado estables y sensibles a los cambios económicos de las sociedades contemporáneas. Por el otro lado, se debe a la menor exigencia normativa que entraña el liberalismo político frente a otras ideologías rivales. Sin embargo, ello conduce a la ambigüedad y la ambivalencia, ya que lo que existe, no es un sólo liberalismo sino diversos liberalismos.

Desde esta perspectiva, Daniel Bell, sostiene que ambas corrientes -liberales y comunitaristas- son liberales, "aunque un extremo del continum se mueva hacia el liberalismo individualista y el otro hacia la definición de la comunidad como [algo] más importante que los derechos individuales" ${ }^{8}$. En este sentido, Concepción Naval menciona que es frecuente el planteamiento acerca de sí el
5 Concepción Naval, Educar Ciudadanos, la polémica liberacomunitarista en educación, España, Ediciones Universidad de Navarra, 1995, p. 66.

6 Ibid., p105

7 Debe advertirse que el liberalismo y comunitarismo, presentan connotaciones muy específicas que difícilmente pueden ser extrapolables al continente Europeo, el cual más bien, ha estado informado de los principios que están en juego, Fernando Bárcena, op. cit. p. 102

8 Daniel Bell, "Las guerras culturales en U.S.A, Comunidad corrección política y multiculturalismo" Claves de la Razón Práctica, Núm. 33, p. 28.

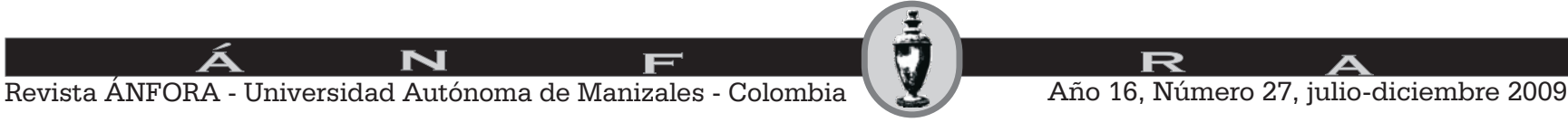


comunitarismo posee realmente una doctrina política, o es más bien, un intento de reunir un conjunto de críticas al liberalismo desde muy diversos ángulos. ${ }^{9} \mathrm{La}$ revisión de cada una de las posturas reclamaría un desarrollo extensivo que no es posible realizar aquí dada la gran cantidad de bibliografía existente y el objetivo concreto de este trabajo. No obstante, brindaremos las líneas generales sobre las cuales se desarrollan el debate entre la corriente liberal y la comunitarista.

Algunos de los exponentes que se distinguen dentro de la corriente liberal son John Rawls, Ronald Dworkin, Robert Nozick, Murray Rothbard. Esta corriente se presenta como una teoría de los derechos, establecida sobre una antropología de corte individualista. Concepción Naval afirma que la hipótesis liberal apunta a la constitución de un individuo separado, existe como un todo completo en sí mismo, que busca maximizar sus ventajas racionales, sin que éstas sean resultado, fundamentalmente, de influencias, experiencias, contingencias y normas propias del contexto social y cultural ${ }^{10}$

Para el liberalismo el individuo tiene prioridad sobre la sociedad. En tanto que ciudadanos, los individuos deben ser plenamente soberanos, y en tanto que seres humanos, requieren de libertad y seguridad para realizar su propia vida. Para este pensamiento, la ciudadanía es una condición que debe ser mantenida y protegida. La política aquí sólo tiene un carácter instrumental, es decir, se pone al servicio exclusivo de proteger a los ciudadanos en el ejercicio de sus derechos. Para esta corriente existe una prioridad ontológica de los derechos sobre las preferencias, ya que los derechos no pueden ser alienados incluso aunque los titulares lo consientan bajo el pretexto de que resultará para ellos un aumento de bienestar o de su satisfacción. ${ }^{11}$ Los derechos de los ciudadanos así, son importantes porque capacitan al individuo a autoprotegerse del gobierno y avanzar en las libertades e intereses individuales y privados.

De acuerdo con esta tradición filosófica, el respeto por los derechos del individuo y el principio de neutralidad política, es el patrón de legitimidad de las democracias constitucionales. En tanto que individuos, los ciudadanos son poseedores de derechos morales, cuyo reconocimiento sirve para limitar la intervención de los otros y del Estado en esfera de la vida privada.$^{12}$

9 Cfr. Concepción Naval op. cit. 98.

10 Ibid. p. 74.

11 Ibid. P. 74-75.

12 Fernando Barcena op.cit. 115-117. 
El papel del Estado en esta corriente no es formar a ciudadanos virtuosos, ni proveer fines particulares. Ni incluso proponer una concepción sustancial de la vida buena, sino sólo garantizar las libertades políticas y civiles fundamentales de tal forma, que cada individuo pueda escoger libremente los fines que se ha fijado en relación con su concepción sobre el bien. El Estado liberal busca así, proporcionar un marco neutro dentro del cual la gente pueda hacer sus propias elecciones. Es decir, respeta la multiplicidad de valores e ideologías, en la medida en que éstas sean compatibles con los principios de justicia. Sus valores son procedimentales, para permitir la coexistencia de otras concepciones diferentes impidiendo que el uso que unos hacen de su libertad dañe a los otros. ${ }^{13}$

La asunción de una parte o de la totalidad de las obligaciones cívicas sólo es una cuestión de elección individual, ya que el ciudadano tiene el derecho de elegir el grado de compromiso en los asuntos cívicos y políticos. La ciudadanía es solamente la adquisición de un estatus y alude a las relaciones formales entre el individuo y el Estado dentro de un marco jurídico. La ciudadanía es una cuestión legal y formal.

Esta visión de la ciudadanía ha sido criticada por varios motivos. Se basa en el principio de exclusión de los no ciudadanos, lo cual implica una separación problemática entre los derechos del individuo y los del ciudadano. Va en contra del fenómeno mundial del multiculturalismo. Y por último, la igualdad de derechos que propone esta visión, no se aplica en la esfera económica dentro de la cual, la libertad se concibe como el derecho a acumular la riqueza de acuerdo con el propio interés y talentos. Esto produce una mayor desigualdad social, provocando una relación inversa entre la igualdad política y la igualdad económica $^{14}$

En contraste los críticos comunitaristas como M. Sandel, A. MacIntryre, Charles Taylor y M. Walzer, R.M Unger, J. Finnis, A. Etzioni, tienen como preocupación central, la importancia de la comunidad en la que el individuo vive. El comunitarismo señala que es justamente, la comunidad en la cual la gente vive la que proporciona las fuentes culturales para que los individuos comprendan tanto el valor de los diferentes modos de vida como así mismos. El objetivo del movimiento comunitario es enunciar una nueva teoría combinando estrechamente la filosofía moral y filosofía política. Esta teoría se elaboró a
13 Concepción Naval, op. cit. p.81.

14 Cfr.Fernando Barcena, El oficio, op.cit., p. 116.

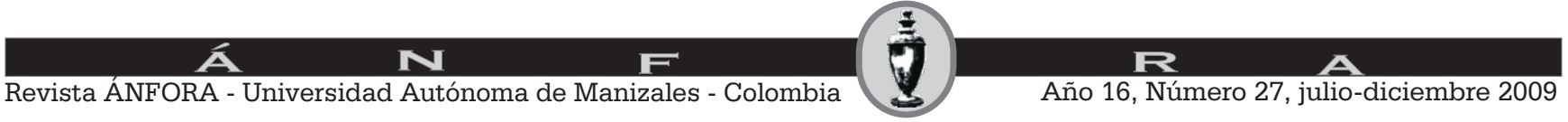


partir de los años ochenta en parte debido a la situación particular de los Estados Unidos donde se destaca la inflación de la política de los derechos por la disgregación de las estructuras sociales, la crisis del Estado-Providencia y la emergencia de la problemática multiculturalista. ${ }^{15}$

El comunitarismo propone que el individuo es un ser esencialmente social. Es constitutivo de su propia autoidentidad el establecimiento de un conjunto de lazos sociales, compromisos y roles comunes. La determinación de cómo deberíamos vivir depende del tipo de relaciones que se sostienen en el ámbito comunitario. La gama de derechos que se despliegan, están en función del sujeto y la comunidad heredera de una tradición moral.

De acuerdo con ello, el comunitarismo cuestiona al liberalismo, debido a esto no comprende del todo la relación entre el individuo y la sociedad. Particularmente, menciona que ignora el alcance formativo que tienen las sociedades. Otro de los aspectos que critica es que el individualismo que plantea el liberalismo es asocial, fomenta y promueve un entendimiento particular entre los individuos y su comunidad. La sociedad es utilizada como una aventura cooperativa para perseguir la ventaja individual ${ }^{16}$ Bajo esta óptica, los comunitaristas afirman que el liberalismo desatiende y hace desaparecer a las comunidades que son elemento fundamental e irremplazable de la existencia humana.

Para esta corriente el hombre se define ante todo como un animal social y político. A partir de ello, la igualdad es definida no como aquello que queda del individuo, sino que es aquello, que resulta de la libre expresión de las identidades constituidas y situadas en su contexto. Así, los derechos no son atributos universales y abstractos, producto de una naturaleza distinta al estado social caracterizado por un dominio autónomo, sino es la expresión de valores propios de las colectividades o de los grupos diferenciados ${ }^{17}$. Del comunitarismo se deriva la creencia de que el desarrollo de la personalidad humana como objetivo principal de la educación, no puede lograrse al margen de la comunidad y los vínculos sociales que los ciudadanos realizan en contextos particulares. La educación dentro de una comunidad y el desarrollo de la civilidad, exigen también organizar la práctica educativa de acuerdo con un concepto de libertad orientado al logro de la felicidad pública. ${ }^{18}$ Para salvar la fragilidad de la vida cívica en la democracia, los comunitaristas proponen
15 Cfr.Concepción Naval, Educar ciudadanos, op. cit. 72.

16 Ibid., p. 90-91

17 Ibid., p. 100.

18 Fernando Barcena op.cit., p. 130.

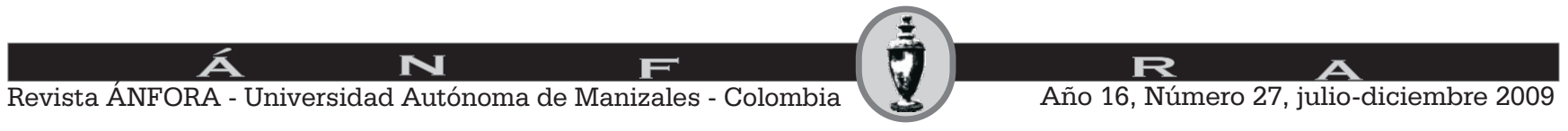


basar las actividades formativas en una idea normativa de la educación, concebida como actividad intrínsecamente moral.

Así sostienen que la ciudadanía no es meramente la adquisición de un título o una condición jurídica, sino una práctica de compromiso orientada a la participación en el ámbito público, la formación de virtudes y la articulación moral del bien público. Esta corriente ve a la comunidad como una fuente de valores, deberes y virtudes sociales en los que se debe educar cívicamente al individuo.

\section{Educar a la ciudadanía}

Frente a este escenario se abre la interrogante de formar cívicamente al ciudadano dando prioridad a la comunidad, su integración y los valores, o la adquisición de una ciudadanía que se base únicamente en la promoción de derechos y obligaciones que el marco jurídico del Estado otorga.

Adoptar un punto de vista individualista o comunitario tiene indudablemente sus consecuencias teóricas y prácticas. No obstante, combatir los excesos del individualismo en nombre de una sociedad, para proponer valores de lo colectivo, es descuidar las razones de la educación ciudadana, es saltar de uno a otro extremo, sin centrarse verdaderamente en el preciso significado de ésta. Su propósito no es la persecución de la verdad, sino más bien la formación de los individuos que pueden mantener su comunidad política y conducir efectivamente sus vidas dentro de ella.

Sin duda, la educación para la ciudadanía no debe ser vista sólo como una condición jurídica, sino que debe apoyarse en una serie de valores que le den sustento para que la democracia se integre no sólo como forma de gobierno, sino también, como forma de vida. Formar al individuo para autoprotegerse del gobierno y avanzar en las libertades e intereses individuales es central, pero no es suficiente. El ciudadano ha de involucrarse en la esfera pública con conocimientos, valores y destrezas que le permitan desarrollar un sentido de lealtad y responsabilidad hacia la comunidad a la que pertenece. En este sentido, la educación cívica no debe ser pensada de manera homogénea o universal. Debe estar dada de acuerdo con un orden político particular.

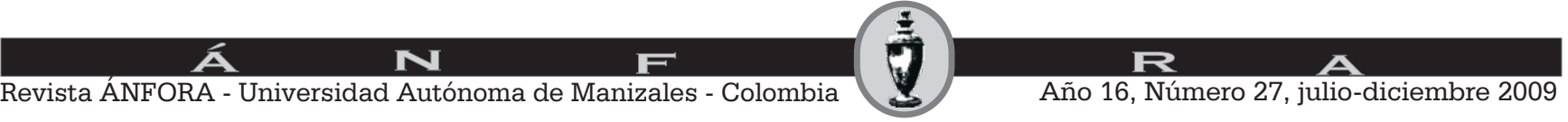


Debe proporcionar conocimiento sobre las instituciones políticas y su historia, iniciación en las prácticas, los derechos y deberes de una comunidad política, el desarrollo de las competencias y virtudes necesarias, el cultivo de las sensibilidades y actitudes tales como el respeto y la solidaridad. Lo cual implicaría una cuidadosa conversación no ideológica, ni doctrinaria, que refleje las tensiones entre razón y la experiencia, el individuo y la comunidad. ${ }^{19}$

En esa medida el aprendizaje de la democracia cobra relevancia si ésta no sólo se concibe como una forma de gobierno, sino como una forma de vida, como una fórmula singular de convivencia social que asume a la vez a los valores democráticos como referentes válidos para la convivencia en general y no sólo para las relaciones público políticas. ${ }^{20}$ De acuerdo con lo anterior, el objetivo de la educación cívica es también, contribuir en el proceso de construcción de una cultura política democrática, donde la participación electoral sea parte de la misma. Así pues, la educación cívica no se agota en cruzadas de capacitación electoral, ni en modificaciones del currículum escolar o la divulgación de los valores democráticos, su campo de acción es más integral y de carácter estructural, es decir, tiene que ver con un proceso de socialización formal e informal que va conformando la cultura política.

La pregunta ¿cómo se aprende a ser ciudadano? puede ser resuelta a través de la enseñanza de educación cívica, no en un sentido curricular, sino como parte de una estrategia más amplia orientada a la construcción de una cultura democrática. Es decir, la educación cívica, no es sólo un aprendizaje académico, es también una práctica cotidiana de valores y actitudes democráticas hacia los procesos e instituciones de una sociedad. En este sentido, la ciudadanía no debe ser vista “..."únicamente como una serie de derechos abstraídos de la práctica cotidiana. No debe plantear la formación de un sujeto moral en abstracto, sino la formación de un sujeto capaz de acción, un actor que conozca, tome conciencia y participe". ${ }^{21}$

Es decir, la educación cívica no debe únicamente capacitar al ciudadano en función de requisitos legales, sino brindarle un marco más amplio que le genere identidad cultural y política, a través de la promoción y enseñanza de los valores y actitudes como la tolerancia, el pluralismo, el consenso, el acuerdo, la participación o el diálogo. La educación cívica es limitada si sólo promueve a la ciudadanía como la adquisición de un estatus o una condición legal que alude
19 Cfr. Concepción Naval, Educar ciudadanos, op. cit. pp. 134-135.

20 Cfr. José Luis Gutiérrez Espíndola, "Educación cívica y construcción democrática en el actual contexto político mexicano", ponencia presentada en el seminariotaller sobre Formación cívica y ética, México, 25 de febrero 1999.

21 Emilio Zebadua González, en el Foro de educación cívica y cultura política democrática, versión estenográfica, de la sesión matutina, Instituto Federal Electoral, México, 4 de noviembre de 1998, p. 5.

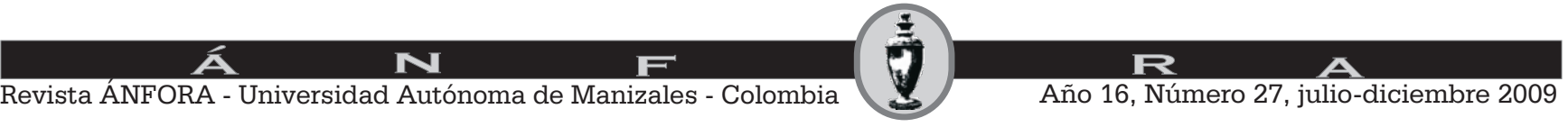


únicamente a las relaciones formales entre individuo y el Estado. Su misión es atender lo anterior y buscar de manera paralela, el establecimiento de un conjunto de lazos sociales y compromisos comunes bajo principios democráticos. Es decir, debe generar prácticas que permitan la participación libre, producto de la autodeterminación de los individuos que deseen involucrarse en la vida pública, la cual sea decidida sin presiones ni imposiciones, a la vez que sea respetuosa del marco jurídico vigente y ajeno a toda forma violenta de relaciones. Igualmente debe fomentar la enseñanza y el ejercicio de los derechos, pero también el cumplimiento de deberes y obligaciones ciudadanas.

Enseñar la democracia es, entonces, enseñar una forma de convivencia, una forma de vida sustentada en valores y actitudes democráticas. La educación cívica requiere ser implementada en las instituciones y en los marcos jurídicos, así como debe ser parte de las actitudes y prácticas cotidianas de los funcionarios públicos, los ciudadanos y de la enseñanza escolar.

De esta forma, el ciudadano no solamente es alguien que elige a los representantes, posee un pasaporte o quien tiene nacionalidad, sino alguien que tiene una conciencia de sí mismo como parte integrante de una comunidad, de una cultura y que esto le genera un sentido de responsabilidad y pertenencia sobre el bien común y el interés general. ${ }^{22}$ La construcción de la ciudadanía a través de la educación cívica requiere de principios, valores, mecanismos e instituciones democráticas, así como disposiciones, destrezas y conocimientos, para participar en la vida pública.

Esta serie de prácticas y aprendizajes tienen que ir acompañadas de mecanismos democráticos e instituciones que permitan el ejercicio de la ciudadanía generando códigos de comunicación compartidos, basados en principios que hagan que la educación cívica sea una práctica cotidiana, que permita al ciudadano involucrase de manera activa, libre y corresponsable en la vida pública.

No se pretende a través de la educación cívica generar ciudadanos adoctrinados o políticos profesionales, sino ciudadanos informados, conocedores de sus derechos y obligaciones, capaces de participar en la vida pública de su país si así lo desean, con un sentido de compromiso ético y
22 Cfr. Concepción Naval, Educar ciudadanos, op. cit. p.186 Véase José Luis Gutiérrez Espíndola, "Educación cívica y construcción democrática en el actual contexto político mexicano", op. cit. pp. 6-10. 
responsabilidad pública en beneficio de su comunidad y del país en general, al tiempo que contribuya a la resolución de los problemas sociales.

No obstante, puede que no baste la enseñanza de conocimientos, prácticas y valores relacionados con la participación y el compromiso cívico, ya que nada garantiza que esto se materialice en actitudes democráticas y en el mejor funcionamiento de las instituciones. Es decir, es difícil saber hasta qué punto la educación cívica puede contribuir a la creación de la confianza social y el compromiso afectivo respecto al sistema político. En todo caso podríamos decir que la educación cívica contribuye a generar ciudadanos potencialmente participativos, informados y conocedores de lo que implica un régimen político democrático con capacidad de participar en la vida pública si así lo desean.

\section{BIBLIOGRAFÍA}

Bárcena Fernando, El oficio de la ciudadanía, Paídos, Buenos Aires, 1997.

Daniel Bell, "Las guerras culturales en U.S.A, Comunidad corrección política y multiculturalismo" Claves de la Razón Práctica, Núm. 33, p. 28.

Suzán Reed Eric, "El concepto de educación cívica. Su vigencia a la luz de otras denominaciones potenciales", XI Conferencia Iberoaméricana de Ministros de Educación, Gobernabilidad democrática y gobernabilidad de los sistemas educativos, 24 y 25 de septiembre de 1996, Concepción Chile.

Naval Concepción, Educar Ciudadanos, la polémica libera-comunitarista en educación, España, Ediciones Universidad de Navarra, 1995.

Gutiérrez Espíndola, José Luis "Educación cívica y construcción democrática en el actual contexto político mexicano", ponencia presentada en el seminariotaller sobre Formación cívica y ética, México, 25 de febrero 1999.

Emilio Zebadua González, en el Foro de educación cívica y cultura política democrática, versión estenográfica, de la sesión matutina, Instituto Federal Electoral, México, 4 de noviembre de 1998.

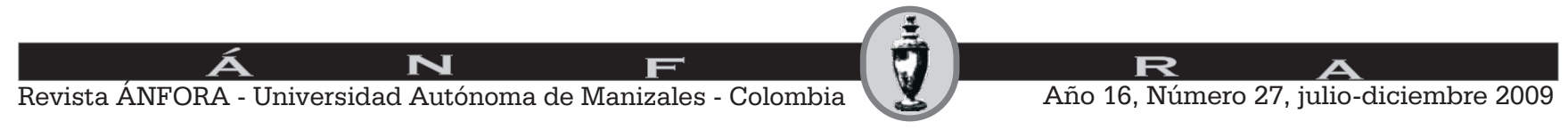

